\title{
Einfluß von Endoxan (Cyclophosphamid) und 6-Mercaptopurin auf den Nuclein- säure-Stoffwechsel der Rattenmilz während der Synthese von Antikörpern gegen Brucella abortus*
}

\author{
H. KRöger und D. Papanastasiu \\ Biochemisches Institut der Universität Freiburg im Breisgau \\ J. Potel \\ Asta-Werke, Chemische Fabrik, Brackwede/W. \\ (Z. Naturforsch. 23 b, 73-77 [1968] ; eingegangen am 30. Mai 1967)
}

1. Endoxan hemmt, insbesondere während der Erstimmunisierung, die Ausbildung von Antikörpern gegen Brucella abortus; 6-Mercaptopurin hat einen weitaus geringeren Effekt.

2. Beide Substanzen vermindern die DNS-Synthese in der Rattenmilz erheblich, die der RNS hingegen nur wenig.

3. Die Induktion der Tyrosin- $a$-Ketoglutarat-Transaminase in der Rattenleber wird durch Endoxan nicht gestört.

4. Diese Ergebnisse lassen den Schluß zu, daß bei der Erstimmunisierung zur Ausbildung der Antikörper eine DNS-Synthese unerläßlich ist.

In der vorangehenden Arbeit berichten wir über den Nucleinsäure-Stoffwechsel, der sich in der Rattenmilz vollzieht während der Erst- und Zweitimmunisierung mit Brucella abortus ${ }^{1}$. In beiden Phasen der Antikörper-Bildung fanden wir die DNS * *-Synthese beträchtlich aktiviert, die RNS-Synthese hingegen nur wenig erhöht.

Bekanntlich wird die Antikörper-Synthese durch zahlreiche Substanzen beeinflußt ${ }^{2}$. STENDER und Mitarb. $^{3,4}$ sowie Potel und Brock ${ }^{5}$ stellten z. B. fest, daß Endoxan die Antikörper-Bildung gegen Brucella abortus stark hemmt, und zwar geschieht dies zu jedem Zeitpunkt der Synthese.

6-Mercaptopurin, ebenfalls ein Antimetabolit der Antikörper-Synthese ${ }^{2}$, hemmt - im Gegensatz zu Endoxan - nur während der induktiven Phase ${ }^{6}$.

In dieser Arbeit beschäftigen wir uns mit der Wirkung von Endoxan und 6-MP auf den NucleinsäureStoffwechsel der Rattenmilz während der Synthese von Antikörpern gegen Brucella abortus.

\section{Methoden}

\section{Immunisierung}

Die Immunisierung der Tiere wurde wie schon beschrieben durchgeführt ${ }^{1}$. Der Antikörper-Titer wurde

\footnotetext{
* Uber einen Teil dieser Untersuchungen wurde auf der Arbeitstagung der Deutschen Gesellschaft für Hygiene und Mikrobiologie, Mainz 1966, berichtet.

**Abkürzungen: AMP = Adenosinmonophosphat; DNS = Desoxyribonucleinsäure; GMP = Guanosinmonophosphat; $\mathrm{IMP}=$ Inosinmonophosphat; 6-MP = 6-Mercaptopurin; RNS $=$ Ribonucleinsäure.

1 H. Kröger u. D. Papanastasiu, Z. Naturforschg., in Vorbereitung.
}

mittels Bakterien-Agglutinations-Reaktion und Wachstumsprobe bestimmt ${ }^{5}$.

\section{Injektion von Endoxan und 6-Mercaptopurin}

Endoxan wurde den Ratten intraperitoneal injiziert an drei aufeinander folgenden Tagen, jeweils $25 \mathrm{mg} / \mathrm{kg}$ Körpergewicht. Gleichzeitig mit der zweiten EndoxanInjektion wurde auch das Antigen verabreicht.

6-MP wurde intravenös appliziert, ebenfalls an drei aufeinander folgenden Tagen, jeweils $20 \mathrm{mg} / \mathrm{kg}$ Körpergewicht. Das Antigen wurde wieder gleichzeitig mit der zweiten 6-MP-Injektion gegeben.

\section{Isolierung der Nucleinsäuren}

Bei der Erstimmunisierung erhielten die Tiere intravenös $36 \mathrm{Stdn}$. nach der Antigen-Gabe $10 \mu \mathrm{C} / 100 \mathrm{~g}$ Körpergewicht ${ }^{3} \mathrm{H}$-Cytidin bzw. ${ }^{3} \mathrm{H}$-Thymidin. Bei der Zweitimmunisierung wurde das ${ }^{3} \mathrm{H}$-Cytidin $36 \mathrm{Stdn}$., das ${ }^{3} \mathrm{H}$-Thymidin $48 \mathrm{Stdn}$. nach der Antigen-Gabe verabreicht. Jeweils 20 Min. nach der Injektion wurden die Tiere getötet und die Milzen entnommen. Die Isolierung der Nucleinsäuren ist in der vorangehenden Arbeit besprochen worden ${ }^{1}$.

Es ist jeweils die einfache Standardabweichung angegeben.

\section{Enzym-Induktion}

Diese wurde in der von $\mathrm{K}_{\text {RöGER }}$ und $\mathrm{G}_{\text {REUER }}{ }^{7}$ beschriebenen Weise durchgeführt.

2 G. H. Hitchings and G. B. Elion, Pharmakol. Rev. 15, 365 [1963].

3 H. S. Stender, D. Strauch u. H. Winter, Strahlentherapie 115, 175 [1961].

4 H. S. Stender, D. Strauch, H. Winter u. W. Textor, Arzneimittel-Forsch. 13, 1031 [1963].

5 J. Potel u. N. Brock, Arzneimittel-Forsch. 15, 659 [1965].

6 R. Schwartz, J. Stack, and W. Dameshek, Proc. Soc. exp. Biol. Med. 99, 164 [1958].

7 H. Kröger u. B. GreUer, Biochem. Z. 341, 190 [1965]. 


\section{Präparate}

${ }^{3} \mathrm{H}$-Cytidin-(G) (spezifische Aktivität 1,9-4 C/ mMol) und ${ }^{3} \mathrm{H}$-Thymidin (6-T) (spezifische Aktivität $1,4-5 \mathrm{C} / \mathrm{mMol}$ ) bezogen wir von The Radiochemical Centre, Amersham, England. 6-Mercaptopurin erhielten wir von Calbiochem, Luzern.

\section{Ergebnisse}

\section{A. Erstimmunisierung}

\section{Einfluß von Endoxan}

Die Ausbildung von Antikörpern gegen Brucella abortus wird durch Endoxan für annähernd 10 Tage vollständig verhindert (s. Abb. 1). Danach steigt der

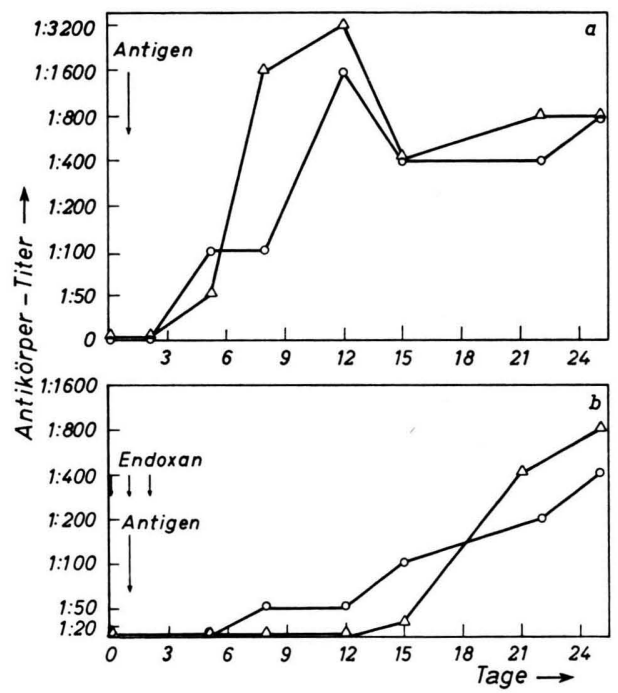

Abb. 1. Einfluß von Endoxan auf die Synthese von Antikörpern gegen Brucella abortus während der Erstimmunisierung. O- $\mathrm{O}$ Bakterienagglutination, $\triangle-\triangle$ Wachstumsprobe, (a) Brucella abortus, (b) Brucella abortus + Endoxan. Angegeben sind Mittelwerte von 10 Tieren. Endoxan-Gabe: dreimal $25 \mathrm{mg} / \mathrm{kg}$ Körpergewicht i.p.

Titer im Serum allmählich an und erreicht etwa am 25. Tag die Kontrollwerte. Aus Tab. 1 ist zu ersehen, wie Endoxan auf den Nucleinsäure-Stoffwechsel der Rattenmilz wirkt. Es schränkt die DNS-Synthese erheblich ein, während die RNS-Synthese weitaus weniger gehemmt wird.

Da das Endoxan die Synthese der Antikörper gegen Brucella abortus verzögert, interessierte uns, ob auch die mit der Antikörper-Bildung verbundene Aktivierung der DNS-Synthese verspätet einsetzt. Wir untersuchten die DNS-Synthese zu verschiedenen Zeiten nach Endoxan- und Antigen-Gabe: etwa

\begin{tabular}{|c|c|c|}
\hline & Ein & \\
\hline & $\begin{array}{c}{ }^{3} \mathrm{H} \text {-Thymidin } \\
\text { in DNS } \\
{[\mathrm{IpM} / \mathrm{mg} \mathrm{DNS}]}\end{array}$ & $\begin{array}{c}\text { 3H-Cytidin } \\
\text { in RNS } \\
{[\mathrm{IpM} / \mathrm{mg} \text { RNS }]}\end{array}$ \\
\hline $\begin{array}{l}\text { Kontrolle } \\
\text { Kontrolle + Endoxan }\end{array}$ & $\begin{array}{l}4168 \pm 474 \\
1542 \pm 217\end{array}$ & $\begin{array}{l}7176 \pm 458 \\
3587 \pm 1045\end{array}$ \\
\hline $\begin{array}{l}\text { Bruc. abortus } \\
\text { Bruc. abortus } \\
\quad+\text { Endoxan }\end{array}$ & $\begin{array}{c}12200 \pm 2800 \\
1531 \pm 423\end{array}$ & $\begin{array}{l}8471 \pm 797 \\
4390 \pm 1015\end{array}$ \\
\hline
\end{tabular}

Tab. 1. Einfluß von Endoxan auf den Nucleinsäure-Stoffwechsel der Rattenmilz während der Erstimmunisierung. Angegeben sind Mittelwerte von 8-10 Tieren. Endoxan-Gabe: an drei aufeinander folgenden Tagen jeweils $25 \mathrm{mg} / \mathrm{kg}$ Körpergewicht i.p.; Antigen-Gabe: zusammen mit der zweiten Endoxan-Injektion. 36 Stdn. nach dem Antigen erhielten die Tiere i.v. $10 \mu \mathrm{C}{ }^{3} \mathrm{H}$-Thymidin bzw. ${ }^{3} \mathrm{H}$-Cytidin/100 g Körpergewicht; 20 Min. danach wurden sie getötet.

48 Stdn. nach der Antigen-Injektion ist die DNSSynthese nahezu ausgeschaltet (s. Abb. 2). Danach nimmt sie allmählich zu, und 6 Tage nach der Applikation des Antigens liegen Werte vor, die deutlich höher sind als die der Kontrollen.

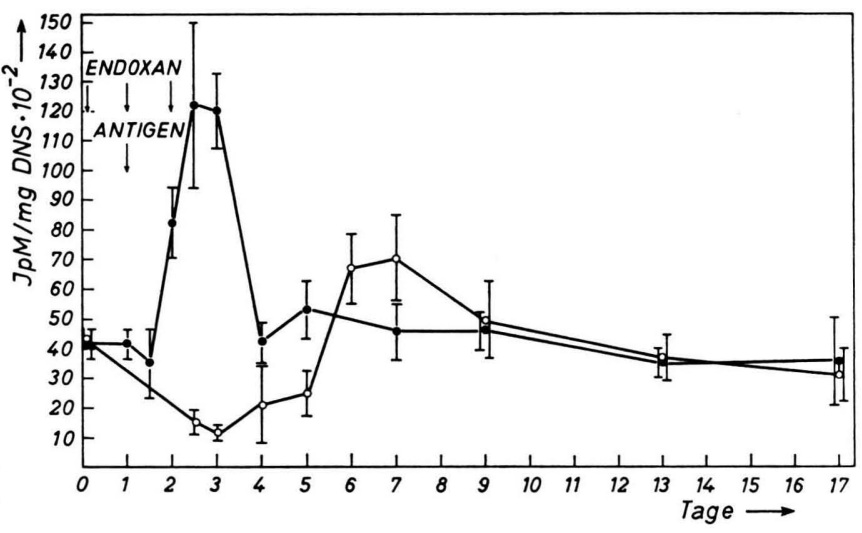

Abb. 2. Einfluß von Endoxan auf die DNS-Synthese der Rattenmilz während der Erstimmunisierung. $\bullet-\bullet$ Brucella abortus, ○-० Brucella abortus + Endoxan. Angegeben sind Mittelwerte von 6-8 Tieren. Endoxan-Gabe: dreimal $25 \mathrm{mg} / \mathrm{kg}$ Körpergewicht i.p. $\mathrm{Zu}$ den angegebenen Zeiten erhielten die Tiere i.v. $10 \mu \mathrm{C}{ }^{3} \mathrm{H}$-Thymidin/100 g Körpergewicht; 20 Min. danach wurden sie getötet.

\section{Einfluß von 6-Mercaptopurin}

Abb. 3 läßt erkennen, daß 6-MP die Ausbildung von Antikörpern gegen Brucella abortus nur geringfügig hemmt. Der Nucleinsäure-Stoffwechsel in der Rattenmilz wird allerdings durch 6-MP ähnlich beeinflußt wie durch Endoxan: die DNS-Synthese wird beträchtlich, die RNS-Synthese aber kaum eingeschränkt (siehe Tab. 2). 


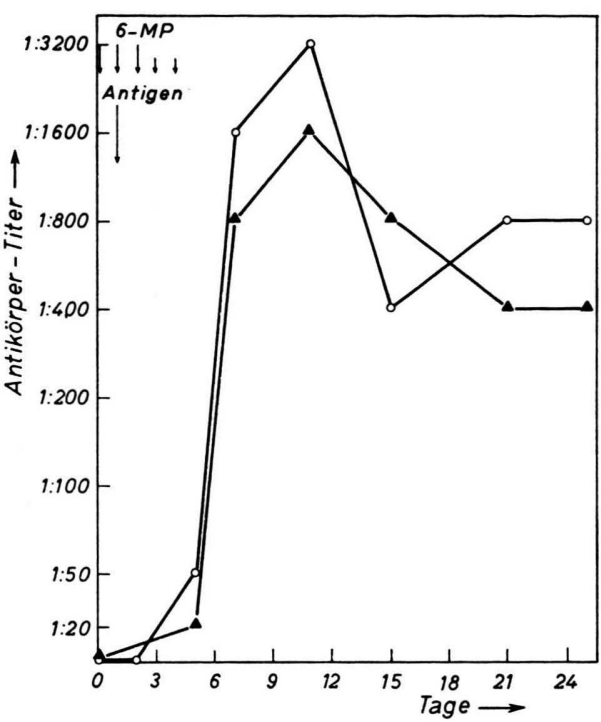

Abb. 3. Einfluß von 6-Mercaptopurin auf die Synthese von Antikörpern gegen Brucella abortus während der Erstimmunisierung. $0-0$ Brucella abortus, $\boldsymbol{\Delta}-\boldsymbol{\Delta}$ Brucella abortus + 6-Mercaptopurin. Angegeben sind Mittelwerte von 8-10 Tieren. Der Antikörper-Gehalt wurde mittels Wachstumsprobe bestimmt. 6-Mercaptopurin-Gabe: dreimal $20 \mathrm{mg}$ und zweimal $10 \mathrm{mg} / \mathrm{kg}$ Körpergewicht i.v.

\begin{tabular}{lcc}
\hline & \multicolumn{3}{c}{ Einbau } \\
& $\begin{array}{c}\text { 3H-Thymidin } \\
\text { in DNS } \\
\text { [IpM/mg DNS }]\end{array}$ & $\begin{array}{c}{ }^{3} \mathrm{H}-\text { Cytidin } \\
\text { in RNS }\end{array}$ \\
{$[\mathrm{IpM} / \mathrm{mg} \mathrm{RNS}]$} \\
\hline Kontrolle & $4168 \pm 474$ & $7176 \pm 458$ \\
Kontrolle + 6-MP & $3568 \pm 1381$ & $5723 \pm 405$ \\
Bruc. abortus & $12200 \pm 2800$ & $8471 \pm 797$ \\
Bruc. abortus + 6-MP & $5816 \pm 1631$ & $7144 \pm 2202$ \\
\hline
\end{tabular}

Tab. 2. Einfluß von 6-Mercaptopurin auf den NucleinsäureStoffwechsel der Rattenmilz während der Erstimmunisierung. Angegeben sind Mittelwerte von 8-10 Tieren. 6-MP-Gabe: an drei aufeinander folgenden Tagen jeweils $20 \mathrm{mg} / \mathrm{kg}$ Körpergewicht i.v.; Antigen-Gabe: zusammen mit der zweiten 6-MP-Injektion. Weiteres s. Tab. 1.

\section{B. Zweitimmunisierung}

\section{Einfluß von Endoxan}

Aus Abb. 4 geht hervor, wie Endoxan bei einer Zweitimmunisierung mit Brucella abortus auf die Antikörper-Synthese einwirkt. Die Verhältnisse unterscheiden sich von denen bei der Erstimmunisierung. Schon direkt nach der Antigen-Gabe nehmen die Antikörper im Serum deutlich zu; allerdings werden die Kontrollwerte - auch in 21 Tagen nicht erreicht. - Den Nucleinsäure-Stoffwechsel der Milz fanden wir bei der Erst- und Zweitimmunisie-

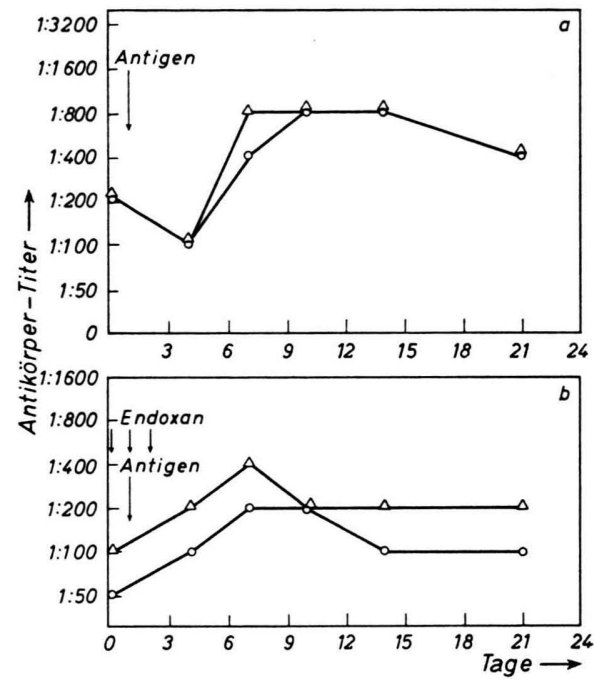

Abb. 4. Einfluß von Endoxan auf die Synthese von Antikörpern gegen Brucella abortus während der Zweitimmunisierung. $0-0$ Bakterienagglutination, $\triangle-\triangle$ Wachstumsprobe, (a) Brucella abortus, (b) Brucella abortus + Endoxan. Angegeben sind Mittelwerte von 8-10 Tieren. Endoxan-Gabe: dreimal $25 \mathrm{mg} / \mathrm{kg}$ Körpergewicht i.p.

rung durch Endoxan recht ähnlich beeinflußt. Tab. 3 zeigt, daß auch bei der Zweitimmunisierung die DNS-Synthese stark gesenkt, die RNS-Synthese hingegen weniger verändert wird.

\begin{tabular}{lcc}
\hline & \multicolumn{2}{c}{ Einbau } \\
& $\begin{array}{c}\text { 3H-Thymidin } \\
\text { in DNS }\end{array}$ & $\begin{array}{c}\text { 3H-Cytidin } \\
\text { in RNS }\end{array}$ \\
& {$[\mathrm{IpM} / \mathrm{mg}$ DNS $]$} & {$[\mathrm{IpM} / \mathrm{mg} \mathrm{RNS}]$} \\
\hline Kontrolle & $3170 \pm 1071$ & $6417 \pm 836$ \\
$\begin{array}{l}\text { Bruc. abortus } \\
\text { Bruc. abortus } \\
+ \text { Endoxan }\end{array}$ & $12166 \pm 1938$ & $8047 \pm 943$ \\
\hline
\end{tabular}

Tab. 3. Einfluß von Endoxan auf den Nucleinsäure-Stoffwechsel der Rattenmilz während der Zweitimmunisierung. Angegeben sind Mittelwerte von 8 Tieren. Endoxan- und AntigenGabe s. Tab. 1. 36 Stdn. bzw. 48 Stdn. nach dem Antigen erhielten die Tiere i.v. $10 \mu \mathrm{C}{ }^{3} \mathrm{H}$-Cytidin bzw. ${ }^{3} \mathrm{H}$-Thymidin/ 100 g Körpergewicht; jeweils 20 Min. danach wurden sie getötet.

\section{Einfluß von 6-Mercaptopurin}

Die Injektion von 6-MP bei der Zweitimmunisierung mit Brucella abortus bleibt wirkungslos auf die Ausbildung der Antikörper (s. Abb. 5). -

Die Untersuchung des Nucleinsäure-Stoffwechsels der Milz verlief wie erwartet: auch während der Zweitimmunisierung vermindert 6-MP die DNSSynthese; wiederum wird der RNS-Stoffwechsel kaum berührt (s. Tab. 4). 


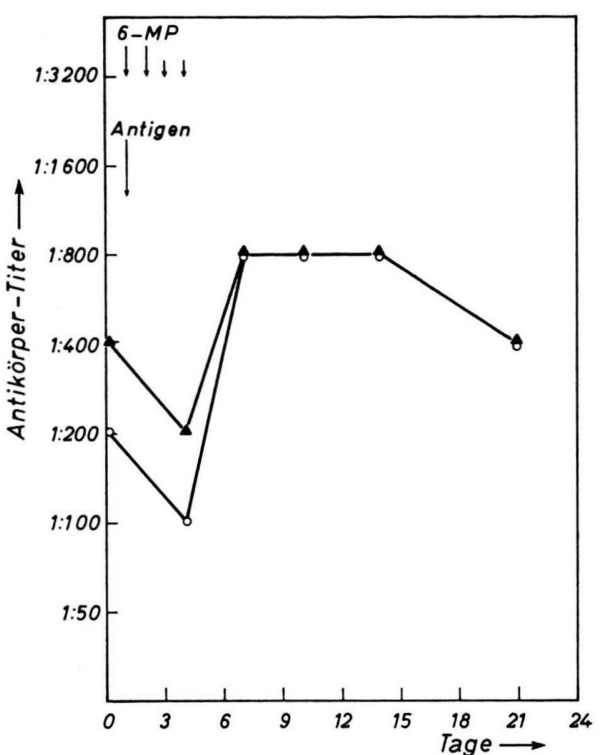

Abb. 5. Einfluß von 6-Mercaptopurin auf die Synthese von Antikörpern gegen Brucella abortus während der Zweitimmunisierung. $\bigcirc-\circ$ Brucella abortus, $\boldsymbol{\Delta}-\boldsymbol{\Delta}$ Brucella abortus + 6-Mercaptopurin. Angegeben sind Mittelwerte von 8 Tieren. Der Antikörper-Gehalt wurde mittels Wachstumsprobe bestimmt. 6-Mercaptopurin-Gabe: dreimal $20 \mathrm{mg}$ und zweimal $10 \mathrm{mg} / \mathrm{kg}$ Körpergewicht i.v.

\begin{tabular}{lrc}
\hline & \multicolumn{3}{c}{ Einbau } \\
& $\begin{array}{c}{ }^{3} \mathrm{H}-\text { Thymidin } \\
\text { in DNS } \\
{[\mathrm{IpM} / \mathrm{mg} \text { DNS }]}\end{array}$ & $\begin{array}{c}{ }^{3} \mathrm{H}-C y t i d i n \\
\text { in RNS }\end{array}$ \\
{$[\mathrm{IpM} / \mathrm{mg} \mathrm{RNS}]$} \\
\hline Kontrolle & $3170 \pm 1071$ & $6417 \pm 836$ \\
Bruc. abortus & $12166 \pm 1938$ & $8047 \pm 943$ \\
Bruc. abortus + 6-MP & $6098 \pm 1588$ & $6385 \pm 719$ \\
\hline
\end{tabular}

Tab. 4. Einfluß von 6-Mercaptopurin auf den NucleinsäureStoffwechsel der Rattenmilz während der Zweitimmunisierung. Angegeben sind Mittelwerte von 8 Tieren. 6-MP- und Antigen-Gabe s. Tab. 2. Weiteres s. Tab. 3.

\section{Diskussion}

Unsere Untersuchungen zeigen, daß Endoxan bei Ratten während der Erstimmunisierung mit Brucella abortus die Ausbildung von Antikörpern erheblich einschränkt. Auch die DNS-Synthese in der Milz ist während dieser Periode stark gehemmt. Erst wenn die Wirkung des Endoxans auf die DNS-Synthese abklingt, lassen sich im Serum Antikörper nachweisen. Die Ursache der verminderten DNS-Synthese

8 G. Palme, E. Liss, K. Oeff u. A. Platis, ArzneimittelForsch. 13, 1034 [1963].

9 J. S. Salser, D. J. Hutchison, and M. E. Balis, J. biol. Chemistry 235, 429 [1960]. ist noch unbekannt. Auch Palme et al. ${ }^{8}$ beobachteten, daß in proliferierenden Geweben die DNSSynthese durch Endoxan gestört wird.

6-MP, das in der von uns verwandten Dosis die Ausbildung von Antikörpern gegen Brucella abortus während der Erstimmunisierung nur wenig herabsetzt, wirkt auf die DNS-Synthese in der Milz ähnlich wie Endoxan; der Effekt ist lediglich schwächer. Über den Mechanismus, der durch 6-MP ausgelöst wird, können wir noch nichts aussagen. In anderen Systemen hat man Beweise dafür, daß durch 6-MP die Überführung vom IMP zu AMP bzw. GMP verhindert wird ${ }^{9-11}$. Aus den bisher vorliegenden Ergebnissen darf geschlossen werden, daß die DNSSynthese unerläßlich ist, damit Antikörper-bildende Zellen entstehen können. Es bleibt fraglich, ob die DNS-Synthese lediglich der Zellvermehrung dient, oder ob sie noch eine andere Funktion wahrnimmt.

Bei der Zweitimmunisierung wird die AntikörperSynthese durch Anwesenheit von Endoxan nur wenig unterdrückt. Das deutet darauf hin, daß in diesem Zeitraum die DNS-Synthese bloß bedingt notwendig ist. Vermutlich sind kompetente Zellen vorhanden, die, angeregt durch das Antigen, direkt die spezifische Messenger-RNS bilden können.

Ein Befund sei hier noch angefügt. Es blieb zu klären, ob auch die Synthese der Messenger-RNS durch Endoxan und 6-MP veränderlich sei. Wir haben in früheren Untersuchungen schon festgestellt, daß 6-MP - auch in sehr hohen Dosen - die Induktion von Enzymen in der Rattenleber nicht beeinflußt ${ }^{7}$. In diesem Zusammenhang studierten wir

\begin{tabular}{lccc}
\hline & Kontrolle & Induktion & $\begin{array}{c}\text { Induktion } \\
\text { unter Endoxan }\end{array}$ \\
\hline L-Tyrosin & 1,07 & $3,96 \pm 0,76$ & $4,08 \pm 0,58$ \\
Cortison & $(2)$ & $(6)$ & $(8)$ \\
& 1,26 & $6,65 \pm \frac{(4)}{(4)} 1,51$ & $5,84 \underset{(8)}{ \pm} 1,37$ \\
\hline
\end{tabular}

Tab. 5. Einfluß von Endoxan auf die Induktion der Tyrosin- $\alpha$ Ketoglutarat-Transaminase in der Rattenleber. Anzahl der Tiere in Klammern. Endoxan-Gabe: an drei aufeinander folgenden Tagen jeweils $25 \mathrm{mg} / \mathrm{kg}$ Körpergewicht i.p. Induktion: direkt nach der letzten Endoxan-Gabe. $\mathrm{L}$-Tyrosin-Induktion: Die Tiere erhielten i.p. $600 \mathrm{mg} \mathrm{L}$-Tyrosin/kg Körpergewicht; 4. Stdn. danach wurden sie getötet. Cortison-Induktion: Adrenalektomierte Tiere erhielten i.p. $40 \mathrm{mg}$ Cortison $/ \mathrm{kg}$ Körpergewicht ; 4 Stdn. danach wurden sie getötet.

10 A. Hampton, Federat. Proc. 19, 310 [1960].

11 J. D. Davidson, Proc. Amer. Assoc. Cancer Res. 3, 15 [1959]. 
nun noch die Wirkung von Endoxan auf die Induktion der Tyrosin- $\alpha$-Ketoglutarat-Transaminase. Wie Tab. 5 zeigt, bleibt die Induzierbarkeit des Enzyms trotz Endoxan erhalten. Die DNS-abhängige RNSSynthese wird also durch die in dieser Arbeit untersuchten Substanzen nicht gehemmt. Dieses Resultat kann als Bestätigung für unsere Schlußfolgerung gewertet werden: Bei der Erstimmunisierung bedarf es unbedingt der DNS-Synthese, damit Antikörper gebildet werden.

Die Untersuchungen wurden unterstützt durch die Deutsche Forschungsgemeinschaft, das Bundesministerium für Wissenschaftliche Forschung und die Stiftung Volkswagenwerk.

\title{
Über die Funktion der sauren Hefephosphatase
}

\author{
Th. Günther und W. Kattner \\ Physiologisch-Chemisches Institut der Freien Universität Berlin \\ (Z. Naturforsch. 23 b, 77-80 [1968]; eingegangen am 10. Juni 1967)
}

\begin{abstract}
Die auf der Zelloberfläche lokalisierte, dereprimierbare saure Hefephosphatase hat keinen Einfluß auf anaerobe Glykolyse und Wachstumsgeschwindigkeit. Bei Phosphatmangel kann sie der Hefezelle im Medium vorhandene Phosphatester als Phosphatquelle erschließen. Die saure Hefephosphatase ist nicht identisch mit der intrazellulär wirksamen Phosphatase.
\end{abstract}

Die biologische Bedeutung der sauren Phosphatasen ist unklar. Es wurde vermutet, $\mathrm{da}$ ß die saure Hefephosphatase intrazelluläres Glucose-6-phosphat spalten und somit die maximale Glykolyserate begrenzen kann ${ }^{1}$. Nach anderen Untersuchungen an E. coli ist die saure Phosphatase Bestandteil des Zuckertransportsystems ${ }^{2}$. Eine Beteiligung am Phosphattransport der Hefezelle wurde nicht gefunden ${ }^{3,4}$. Da die saure Hefephosphatase ein dereprimierbares Enzym ist ${ }^{5-9}$, hat man die Möglichkeit, durch den Vergleich phosphatasearmer und phosphatasereicher Zellen desselben Hefestammes auf die Rolle der sauren Phosphatase im Stoffwechsel von Hefezellen zu schließen.

\section{Methode}

\section{Züchtung der Zellen}

Wir verwendeten Germaniahefe, Stamm A (Norddeutsche Hefeindustrie). Die Züchtung erfolgte in Erlenmeyer-Kolben im Brutschrank bei $30{ }^{\circ} \mathrm{C}$ unter Begasung mit Luft.

1 L. Kiesow u. S. Döge, Z. Naturforschg. 16 b, 576 [1961]

2 W. Kundig, F. D. Kundig, B. Anderson u. S. Roseman, J. biol. Chemistry 241, 3243 [1966].

3 A. Rothstein u. R. Meier, J. cellular comparat. Physiol. 34, 97 [1949].

4 G. W. F. H. Borst Pauwells u. R. Reijnen, Biochim. biophysica Acta [Amsterdam] 93, 659 [1964].

5 H. Suomalainen, M. Linko u. E. Oura, Biochim. biophysica Acta [Amsterdam] 37, 482 [1960].
J)as Kulturmedium enthielt im $l 3,0 \mathrm{~g}\left(\mathrm{NH}_{4}\right)_{2} \mathrm{SO}_{4}$, $0,25 \mathrm{~g} \mathrm{MgSO}_{4} \cdot 7 \mathrm{H}_{2} \mathrm{O}, 0,25 \mathrm{~g} \mathrm{CaCl}_{2}, 0,4 \mathrm{~g} \mathrm{KCl}, 1 \mathrm{~g}$ Glycin, 1,5 g Hefeextrakt (Difco), $15 \mathrm{mg}$ Penicillin G-Na, $25 \mathrm{mg}$ Streptomycinsulfat und $5 \mathrm{mg}$ Polymyxin $\mathrm{B}$. Die jeweiligen $\mathrm{PO}_{4}$-Konzentrationen sind in den Legenden enthalten.

Bei aerober Züchtung wurden $2 \%$, bei anaerober $5 \%$ Glucose zugegeben. Anaerobe Bedingungen wurden durch Zugabe von $1 \mathrm{mMol} \mathrm{KCN}$ hergestellt. Hierbei wurde das Medium zusätzlich mit 0,05-m. Succinat auf $p_{\mathrm{H}} 5,2$ gepuffert.

\section{Bestimmung der Zellmasse}

Die Bestimmung der Zellmasse in den Kulturen erfolgte durch $20 \mathrm{~min}$ Zentrifugieren bei $1600 \mathrm{~g}$ in geeichten Hämatokritröhrchen oder durch Trübungsmessung bei $405 \mathrm{~nm}$.

\section{Proteinbestimmung}

Die Zellen wurden durch 30 min Erhitzen bei $80{ }^{\circ} \mathrm{C}$ in $1-n$. $\mathrm{NaOH}$ aufgeschlossen. Proteinbestimmung nach Lowry et al. ${ }^{10}$.

${ }^{6}$ C. F. Heredia, F. Yen u. A. Sols, Biochem. biophysic. Res. Commun. 10, 14 [1963].

7 G. Schmidt, G. Bartsch, M. C. Laumont, T. Herman u. M. Liss, Biochem. J. 2, 126 [1963].

8 W. L. McLellan u. J. O. Lampen, Biochim. biophysica Acta [Amsterdam] 67, 324, [1963].

9 R. Weimberg u. W. L. Orton, J. Bacteriol. 89, 740 [1965].

10 O. H. Lowry, N. J. Rosebrough, A. L. Farr u. R. J. Randall, J. biol. Chemistry 193, 265 [1951]. 